\title{
Comparison between Horn and Bow-tie Antennas for Ground Penetrating Radar
}

\author{
Massimiliano Pieraccini, Neda Rojhani, Lapo Miccinesi \\ Department of Information Engineering \\ University of Florence \\ Via Santa Marta, 3 \\ 50139 Firenze, Italy \\ massimiliano.pieraccini@unifi.it
}

\begin{abstract}
Many different antennas are proposed and tested for GPR, but bow-tie or horn antennas are doubtless the most used. Their simple and robust design makes them effective in most applications. The aim of this paper is a direct comparison between a horn and a bow-tie antenna, used in the same operative conditions, i.e. in the same band, with the same transceiver and on the same ground. Although this is a preliminary study based on a very limited set of experimental data, horn antenna appears to have advantages with respect to bow-tie antenna in GPR applications.
\end{abstract}

Keywords-antennas, GPR, radar

\section{INTRODUCTION}

Ground Penetrating Radars (GPR) are popular instruments able to investigate the ground trough electromagnetic waves. They are commonly used for detecting pipes under the floor or the asphalt [1], buried ancient artifacts [2] or parts of ancient buildings [3], as well as in many other applications [4].

A key element of any GPR is the antenna. While the antennas for telecommunications are designed for operating in the free space, the antennas for GPR must operate close or in contact of the ground. This is a strict constraint in their design. As the near field is not in the free space, their performances can vary dramatically in different soils. Furthermore, they have to be well-shielded in the back for avoiding to detect "air events", i.e. targets in the air that can be confused with targets in the subsurface. Many different antennas are proposed and tested for GPR [4], but bow-tie or horn antennas are doubtless the most used [5]-[13]. Their simple and robust design makes them effective in most applications.

Bow-tie antennas need a balanced feeder and a shield in rear, nevertheless they are more popular then horns, probably because they are cheaper, less bulky and they are optimally coupled with the ground in the whole frequency range. Horn are usually not used in contact with the soil/asphalt/concrete/wall under test, but in close proximity [7],[10]-[13]

The aim of this paper is a direct comparison between a horn and a bow-tie antenna, used in the same operative conditions: in the same band, with the same transceiver and on the same ground. The horn antennas were two Schwarzbeck mod. BBHA 9120 A (1201) (Schwarzbeck Mess - Elektronik, Schönau, Germany) designed for operating in free-space in the band $0.8 \mathrm{GHz}-5.2 \mathrm{GHz}$, while the bow-tie antennas were two
SPR 1000 (Florence Engineering, Firenze, Italy) designed for operating in contact with the soil in the $350 \mathrm{MHz}-1.5 \mathrm{GHz}$. The common band we selected was $0.8 \mathrm{GHz}-1.2 \mathrm{GHz}$.

\section{THEORY}

Before the experimental test we tried to theoretically predict the behavior of the two antenna with simplified models. The modeling that follows relies on strongly simplifying assumptions, but its aim is just to identify the physics at the basis of this comparison. A deeper analysis using more sophisticated simulation tools will be the subject of a further work.

A bow-tie antenna well-matched with the ground and with its absorbing material in the rear, can be roughly approximated with a half wavelength dipole. Therefore its the gain in the direction $\vartheta_{b}=\pi / 2$ (see Fig. 1) is $G_{1}=1.643$ [14]

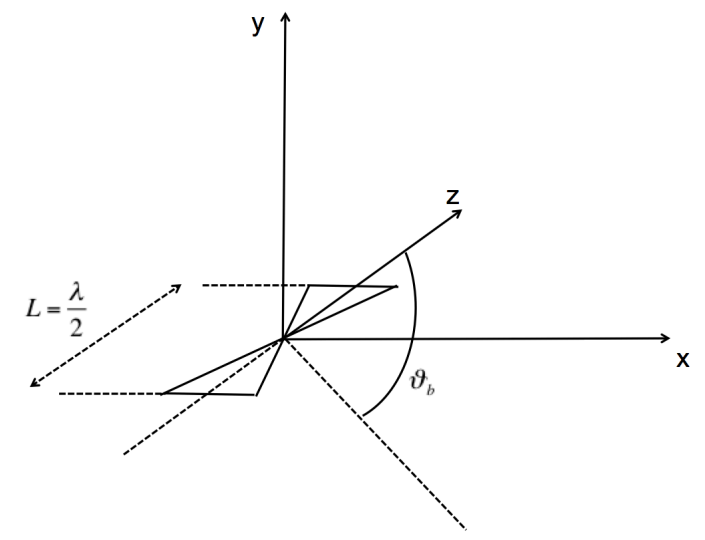

Fig. 1. Bow-tie antenna

The geometry of a horn antenna is shown in Fig. 2. The standard formulas for calculating its radiated pattern assume that it is operating in the free space [14]. As we used the horn in contact with the soil, we made the following (rough) assumptions: 1) the field in the antenna mouth is calculated assuming free space propagation (inside the horn there is air), 2) the antenna mouth is in contact with the soil and so there is a back-reflected wave due to the dielectric change between air and soil, 3) the propagation in front of the mouth takes into account of the soil permittivity. 


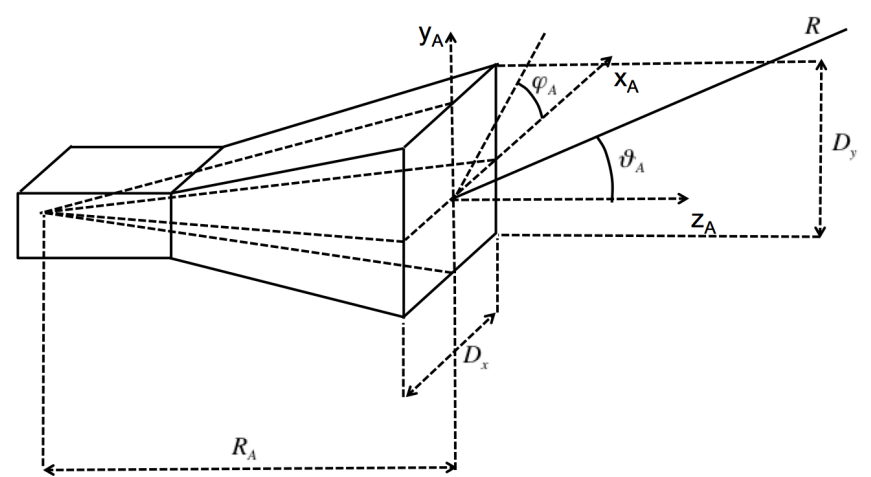

Fig. 2. Horn antenna

Therefore, the integral we used for calculating the radiated was the following

$$
\begin{gathered}
E\left(\vartheta_{A}, \varphi_{A}\right)=\frac{2 \pi}{\lambda}\left(1+\cos \vartheta_{A}\right) \times \\
\times \int_{x_{A}=-\frac{D_{x}}{2}}^{x_{A}=\frac{D_{x}}{2} \int_{y_{A}}=\frac{D_{y}}{2}=\frac{D_{y}}{2}} E_{A}\left(x_{A}, y_{A}\right) e^{j \frac{2 \pi}{\lambda}\left(x_{A} \sin \vartheta_{A} \cos \varphi_{A}+y_{A} \sin \vartheta_{A} \sin \varphi_{A}\right)} d x_{A} d y_{A}
\end{gathered}
$$

The angles $\theta_{A}, \varphi_{A}$, and the distances $R_{A}, D_{x}, D_{y}$ are defined in Fig. 2. $\lambda_{0}$ is the wavelength in the vacuum, $\lambda$ is the wavelength in the medium in front of the antenna.

$$
E_{A}\left(x_{A}, y_{A}\right)=E_{0} \cos \left(\frac{\pi x_{A}}{D_{x}}\right) e^{-j \frac{\pi}{\lambda_{0}}\left(\frac{x_{A}^{2}}{R_{A}}+\frac{y_{B}^{2}}{R_{A}}\right)}
$$

By taking into account of the back-reflected wave, the gain for $\varphi_{A}=\pi / 2$ can be calculated as

$$
G_{2}=\left(1-\left(\frac{\sqrt{\varepsilon_{r}}-1}{\sqrt{\varepsilon_{r}}+1}\right)^{2}\right) \frac{4 \pi\left|E\left(\vartheta_{A}, \pi / 2\right)\right|^{2}}{\int_{\vartheta_{A}=0}^{\vartheta_{A}=\pi / 2} \int_{\varphi_{A}=0}^{\varphi_{A}=2 \pi}\left|E\left(\vartheta_{A}, \varphi_{A}\right)\right|^{2} \sin \vartheta_{A} d \vartheta_{A} d \varphi_{A}}
$$

with $\varepsilon_{r}$ relative permittivity of the soil. The measurement geometry we consider is shown in Fig. 3. The aim is to asses the radar response of a generic point-target of radar-crosssection (RCS) $\sigma$ at depth $z$ between the two antennas. The distance between the antennas centers is $L_{0}$.

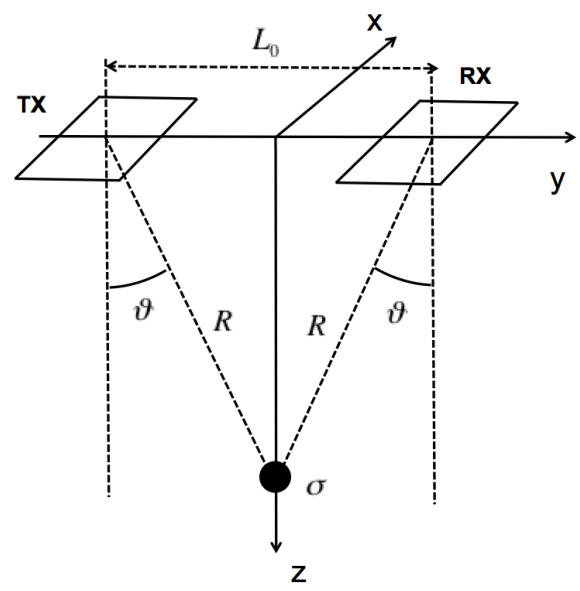

Fig. 3. GPR Geometry
By using the radar equation, the received power is

$$
P_{R X}=P_{T X} \frac{G_{i}^{2}}{4 \pi R^{2}} e^{-\alpha R} \sigma \frac{1}{4 \pi R^{2}} e^{-\alpha R} \frac{\lambda^{2}}{4 \pi}
$$

where

$$
R=\sqrt{\left(L_{0} / 2\right)^{2}+z^{2}}
$$

$\alpha$ is the attenuation coefficient of the soil (at the central frequency of the radar).

Therefore

$$
\frac{P_{R X}}{P_{T X} \sigma}=\frac{G_{i}^{2}}{\left(4 \pi R^{2}\right)^{2}} \frac{\lambda^{2}}{4 \pi} e^{-\alpha(2 R)}
$$

\section{SIMULATIONS}

We simulated the responses of a bow-tie antenna and a horn antenna using the following parameters: relative permittivity $\varepsilon_{r}=4.49$ (see experimental section), central frequency $f_{c}=1.0$ $\mathrm{GHz}$, width of horn $D_{x}=245 \mathrm{~mm}$, height of horn $D_{y}=140 \mathrm{~mm}$, length of bow-tie $R_{A}=185 \mathrm{~mm}$, soil attenuation $\alpha=0.94 \mathrm{~m}^{-1}$ (see experimental section), distance between the centers of antennas $L_{0}=0.48 \mathrm{~m}$. Fig. 3 shows the obtained plot of (5) versus depth $z$. It is interesting to note that the response of the horn antennas appears much higher for depth larger then $0.2 \mathrm{~m}$.

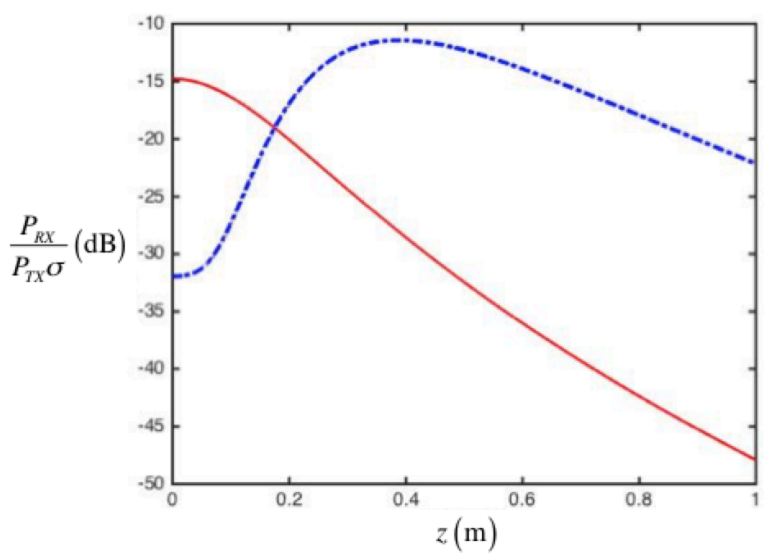

Fig. 3. Response of the bow-tie antennas (Red full line) and of the horn antennas (Blue dotted line)

\section{MEASUREMENT SET-UP}

The experimental setup we used for testing the performances of the two couples of antennas is shown in Fig. 5 . 


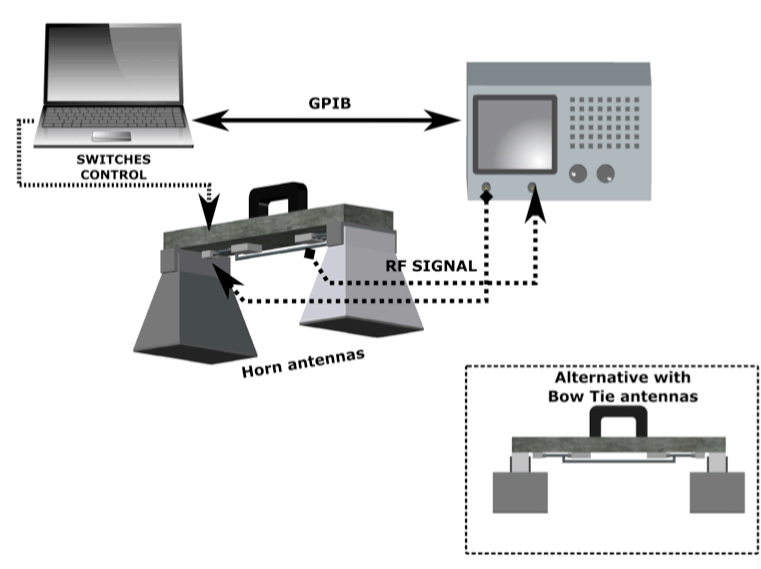

Fig. 5 Experimental setup

A VNA (HP 8753D) operated as Continuous Wave Step Frequency (CWSF) transceiver. It is linked to the radar frontend through microwave cables as shown in Fig. 6. The frontend consists of two wideband amplifiers (respectively in TX and RX channel) with $11 \mathrm{~dB}$ gain, and a pair of single-pole double-throw (SPDT) switches that provides a direct path (through a $-40 \mathrm{~dB}$ attenuator) between the TX and RX channels in order to perform calibrated measurements. At the end of the receiver chain (before the VNA) a Low Pass Filter cuts possible harmonic frequencies that the amplifiers can introduce.

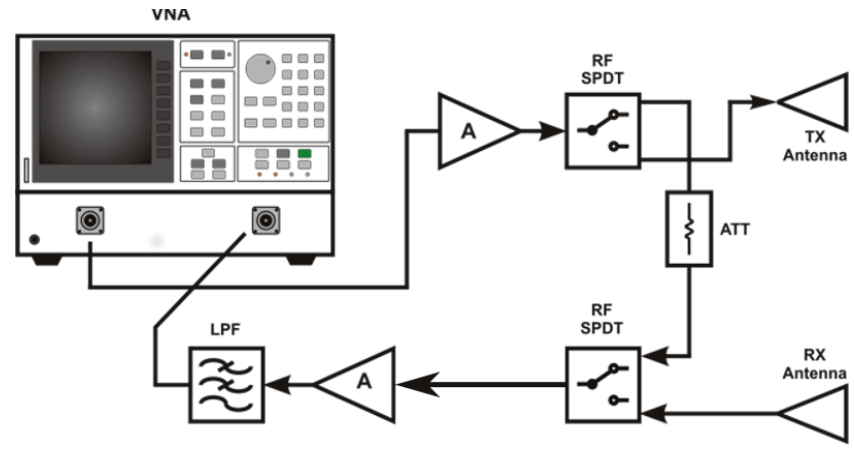

Fig. 6. Radar front-end

Figure 7 is a picture of the two couple of antennas.

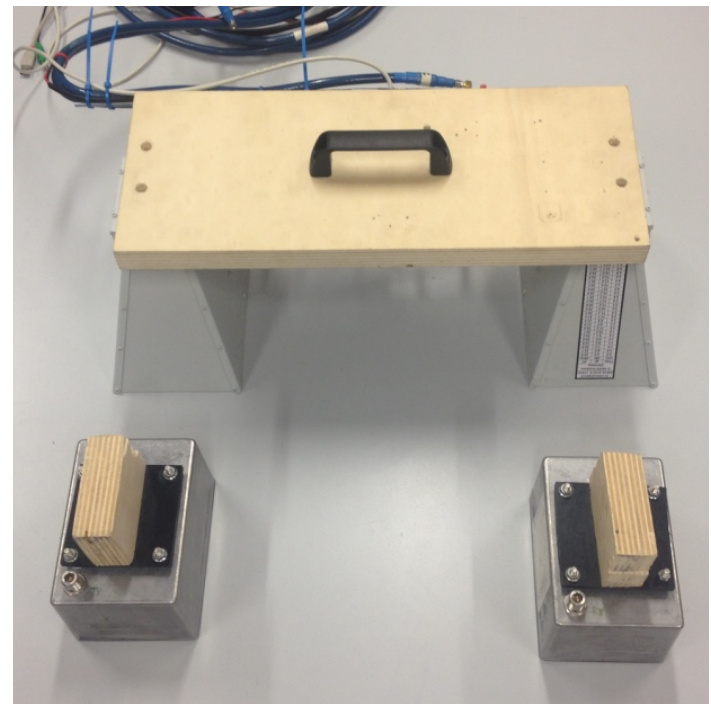

Fig. 7 Picture of the two couples of antennas

\section{MeAsurements RESUlts}

Before to test the performances of the two couples of antennas on the ground we measured the band of the antennas and we estimated the permittivity and the attenuation of the soil (a ground floor, indeed) where the antennas have been used.

With the aim to evaluate the operating bands of the two couples of antenna, we linked them to the VNA and we put them face each other (see Fig. 8 relative to the horn antennas measurement) with a concrete layer interposed for simulating the effect of the contact with a realistic propagation medium

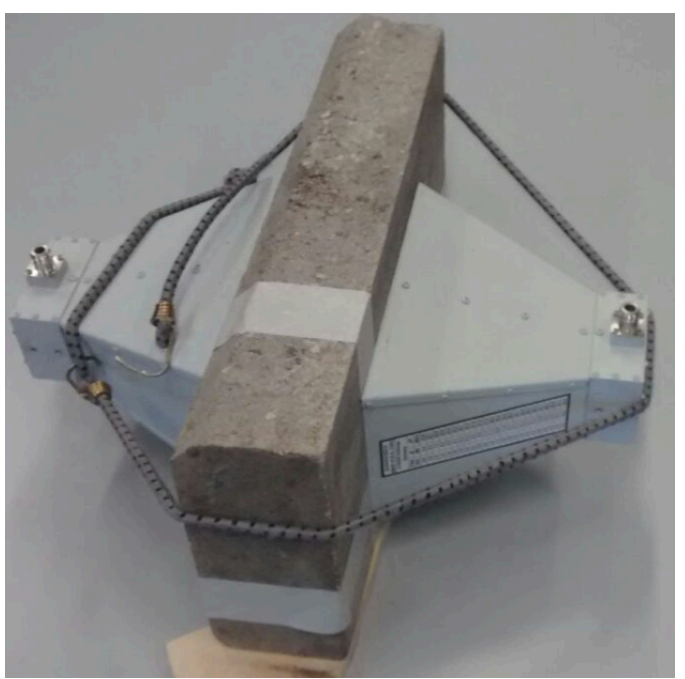

Fig. 8 Picture of the experimental setup for measuring the band of the horn antennas

Fig. 9 shows the measured bands of the bow-tie and the horn. The common band we selected has been $0.8 \mathrm{GHz}-1.2$ GHz. 


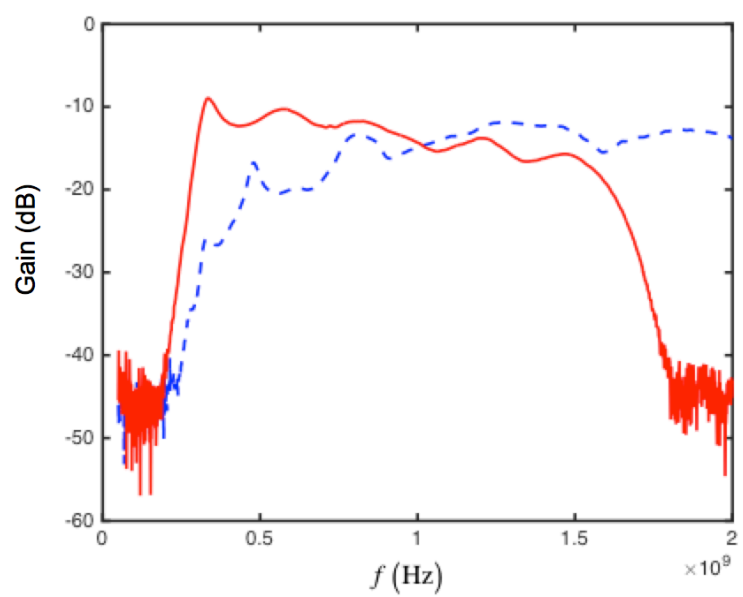

Fig. 9 Measured bands of the bow-tie antennas (Red full line) and of the horn antennas (Blue dotted line)

With the aim of estimate the permittivity of the soil where the antennas has been tested, we performed a measurement with horn antennas in air and a second one with the antennas on the soil. The result of each measurement was a complex array $E_{i}$, whit $i$ frequencies index $\left(1<i<N_{f}\right)$. A Kaiser window (with $\beta=5$ ) has been applied to this array for decreasing the side lobes and than we calculated the Inverse Fast Fourier Trasform (IFFT) with a padding factor $F=100$. The two power plots in time domain (respectively with the antennas in air and with the antennas on the ground) are compared in Fig. 10. The first peak corresponds at the direct path between the two antenna in air and the second one corresponds at the direct path in the soil.

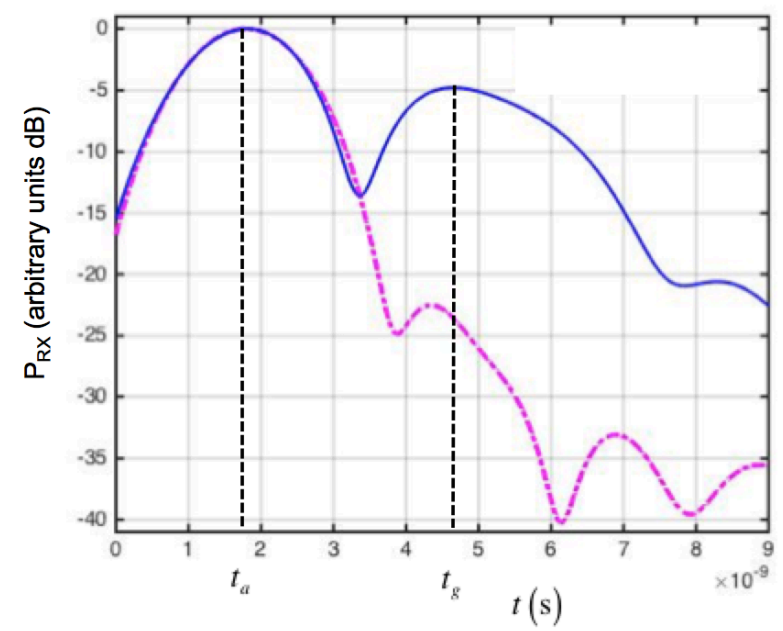

Fig. 10 Power plot in time for horn antennas in air (red dotted line) and on the ground (blue full line)

The $\sqrt{\varepsilon_{r}}$ has been evaluated by using the following relationship

$$
\sqrt{\varepsilon_{r}}=\frac{c}{v}=\left(1+\frac{L_{0}}{c}\left(\frac{1}{t_{g}}-\frac{1}{t_{a}}\right)\right)^{-1}
$$

where $c$ is speed of light in vacuum, $v$ speed of electromagnetic wave in the ground, $t_{a}$ and $t_{g}$ are the time of flight respectively in air and in the ground. The measured value has been $\varepsilon_{r}=4.49 \pm 0.25$.

The attenuation coefficient $\alpha$ has been estimated using the following relationship

$$
\alpha=\frac{1}{D} \ln \left(\frac{P_{a}}{P_{g}}\right)
$$

where $P_{a}$ and $P_{g}$ are the peak amplitude respectively in air and in the ground. The measured value has been $\alpha=(0.94 \pm$ $0.18) \mathrm{m}^{-1}$.

We performed a scan of $3 \mathrm{~m}$ in a zone where there were not evident targets and we plotted the power versus depth both for bow-tie antennas and horn antennas. The obtained plots are shown in Figure 11. They confirm qualitatively the simulated findings. The horn antennas are able to provide a higher signal even if the experimental margin is not as large as in the simulation. But this discrepancy is expected, as in the simulation we considered a single target, while in the experimental case the receiver integrates all the targets inside the TX-RX lobes. Furthermore, we note that the response of the bow-tie antennas is lower for short range contrary to the simulation. It is probably due to the fact that the bow-tie plane is not exactly on the external surface of the metallic case, but a bit inside. So the metallic shield attenuates the lobe at the largest angles.

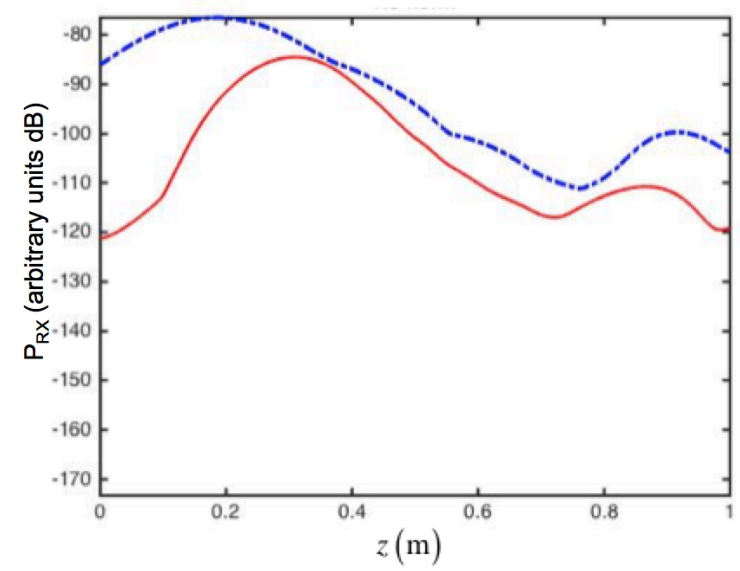

Fig. 11 Measured power plot in depth for horn antennas (blue dotted line) and for bow-tie (red full line)

Finally we tested the two antennas scanning the same track where there were some known targets (three pipes orthogonal to the track at about $20 \mathrm{~cm}$ in depth). The obtained radar images without any focusing or other sophisticated processing are shown in Figure 12 and 13. The circles indicate the position of the pipe under the floor. 
The radar image obtained with the horn antennas is much more clear. This is due both the higher power and to the narrower beam. Nevertheless the horn antennas seems give better images then bow-tie antennas

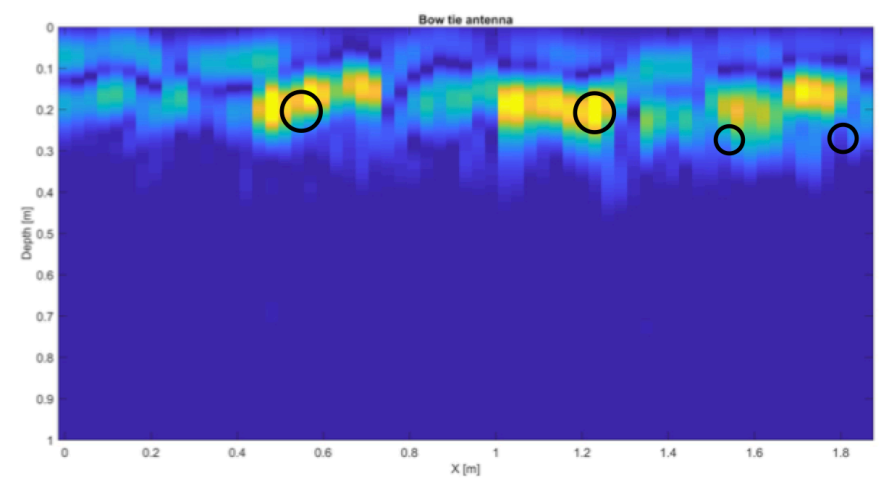

Fig. 12 Radar image using the bow-tie antennas

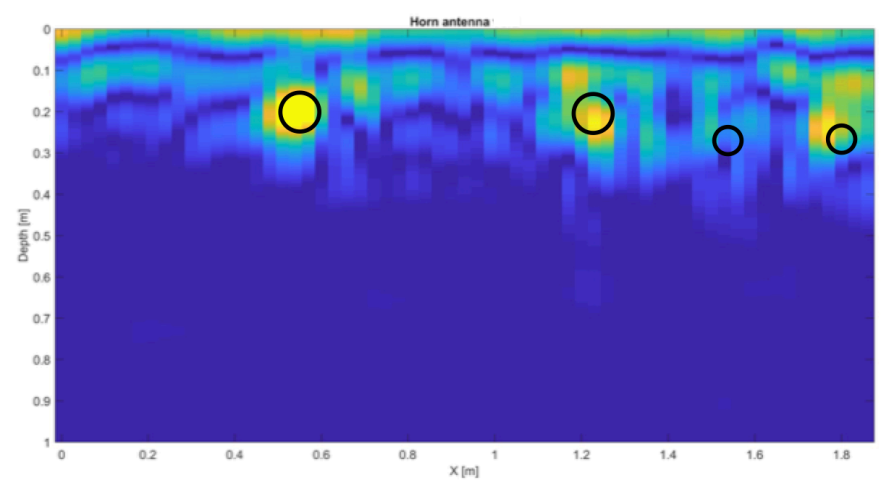

Fig. 13 Radar image using the horn antennas

\section{CONCLUSIONS}

A couple of bow-tie antennas and a couple of horn antennas operating in the same conditions have been compared. Although this is a very preliminary study based on a single set of experimental data and on a strongly simplified theoretical model, horn antennas appear to have advantages with respect to bow-tie antennas in GPR applications. Nevertheless this finding should be confirmed by further studies that should include a deeper analysis carried out using more sophisticated simulation tools and experimental tests on different test sites. Another significant development of this study could be a comparison bow-tie, horn and horn filled with a dielectric. This last solution should improve the coupling between the horn antenna and the ground.

\section{REFERENCES}

[1] C. Windsor, L. Capineri, P. Falorni, S. Matucci, G. Borgioli, "The estimation of buried pipe diameters using ground penetrating radar." Insight-Non-Destructive Testing and Condition Monitoring, Vol. 47, No. 7, pp. 394-399, 2005

[2] L. B. Conyers, "Ground-penetrating radar for archaeology." Altamira Press, 2013.

[3] F. Grasso, G. Leucci, N. Masini, R. Persico. "GPR prospecting in Renaissance and baroque monuments in Lecce (Southern Italy)." In Advanced Ground Penetrating Radar (IWAGPR), 2011 6th International Workshop on (pp. 1-4), June, 2011.

[4] D. J. Daniels, "Ground penetrating radar." John Wiley \& Sons, Inc., 2005 .

[5] S. G. Millard, A. Shaari, J. H. Bungey. "Field pattern characteristics of GPR antennas." NDT \& E International , Vol. 35 No. 7, pp. 473-482 October 2002

[6] F. Parrini, M. Pieraccini, D. Mecatti, D. Dei, G. Macaluso, A. Spinetti, R. Persico, G. Leucci, G. Manacorda, "A reconfigurable stepped frequency GPR (GPR-R): The antenna subsystem. In 2013 7th International Workshop on Advanced Ground Penetrating Radar. July 2013.

[7] F. Parrini, F. Papi, M. Pieraccini. "An ultra high resolution stepped frequency GPR for civil engineering applications." Advanced Ground Penetrating Radar (IWAGPR), 2015 8th International Workshop on. IEEE, 2015.

[8] A. G. Yarovoy, L. P. Ligthart. "Ultra-wideband antennas for ground penetrating radar." Proc. International Symposium on Antennas for Radar Earth Observation, Symposium, Delft University of Technology, The Netherlands. 2000.

[9] G. Chen, R. C. Liu. "A 900MHz shielded bow-tie antenna system for ground penetrating radar." Ground Penetrating Radar (GPR), 2010 13th International Conference on. IEEE, 2010.

[10] B. Panzner, A. Jöstingmeier, A. Omar. "A compact double-ridged horn antenna for ground penetrating radar applications." Microwave Radar and Wireless Communications (MIKON), 2010 18th International Conference on. IEEE, 2010.

[11] T. A. Serdar, A. K. Keskin. "Ultra wide band TEM horn antenna designs for ground penetrating impulse radar." 2012 IEEE International Conference on Ultra-Wideband. IEEE, 2012.

[12] A. G. Emam, A. A. Shaalan, K. F. Ahmed Hussein. "Wideband partially-covered bowtie antenna for ground-penetrating-radars." Progress In Electromagnetics Research Vol. 71, pp. 211-226, 2007

[13] T. Saarenketo, T. Scullion. "Road evaluation with ground penetrating radar." Journal of applied geophysics, Vol. 43. No. 2, pp. 119-138, 2000

[14] C. A. Balanis, "Antenna theory: analysis and design.” John Wiley \& Sons, 2016. 